\title{
Cleaning Calcium Sulfate in Mine Water Membranes
}

\author{
Gregory Gibson, Max Fazel, Stephen P. Chesters \\ Genesys International Ltd. UK \\ 3A Aston Way, Middlewich, Cheshire, CW10 0HS UK
}

\begin{abstract}
Mining industries continue to use large volumes of water to facilitate the extraction of precious metals from rock; resulting in large quantities of waste water. Membrane technologies are capable of producing clean permeate water for reuse onsite, and the concentrate stream can be used for precious metal recovery. Acid mine drainage (AMD) is a problem in most mines due to high levels of pyrite $\left(\mathrm{FeS}_{2}\right)$ within the embedded rock. These AMD waters are often very acidic and have high levels of metals, which if untreated will lead to rapid fouling and scaling of the membrane plant. Calcium Sulfate (Gypsum) scaling is prevalent and can cause catastrophic failure of the membranes. In this paper the authors have investigated the chemistry of $\mathrm{CaSO}_{4}$ scaling. Flat sheet test rigs and 2.5 " membranes were deliberately scaled with simulated mine waters and then different cleaning reagent formulations used to try and remove the calcium sulfate scale. Some new cleaning product formulations, termed Cleaner A and Cleaner B have shown promise in their ability to remove $\mathrm{CaSO}_{4}$ scale with the results presented herein.
\end{abstract}

Keywords: Calcium Sulfate, Mining, Acid Mine Drainage, Reverse Osmosis, Pyrite, Gypsum, Flat Sheet Rig, Membrane Cleaning.

\section{Introduction}

Mining is the fourth largest water consumer with the extraction of precious minerals such as gold $(33,000$ litres/ounce), copper $(18,000$ litres $/ \mathrm{kg})$ and aluminium $(8,000$ litres $/ \mathrm{kg})$ requiring large quantities of water [1].

One of the biggest issues involved with using so much water during the extraction process is a chemical process which leads to what is termed acid mine drainage (AMD). This problem is not a new one, however due to environmental legislation and more stringent regulations, this issue can no longer be ignored. AMD is defined as "the outflow of acidic water from either metal mines or coal mines". AMD waters are low $\mathrm{pH}$, usually with high concentrations of metals; particularly iron, aluminium and manganese, as well as lower concentrations of toxic heavy metals. AMD waters are most commonly found in mines that are abundant in pyrite, which is readily oxidised. Pyrite, also known as "fool's gold" is a mineral belonging to the iron sulfide family $\left(\mathrm{FeS}_{2}\right)[2]$.

Reverse Osmosis (RO) is a technique used in desalination in the treating of waters with high concentrations of salts. $\mathrm{RO}$ is defined as "a process by which a solvent can pass through a porous membrane in the direction opposite to that for natural osmosis when subjected to a hydrostatic pressure greater than the osmotic pressure". Simply put, this is where water with high TDS or ion concentrations have these ions removed via pressure, producing water that is suitable for drinking and other purposes where a low ion concentration is important. With so much water being used daily in the mining industry, RO is a technique that could be employed to help recycle some of the waste water associated with mining. One of the fundamental issues with RO technologies is the ability to keep membranes clean from both fouling and scaling, both of which have the potential to be catastrophic to a plant unless proper pre-treatment is implemented. This is even more of an issue in mining as the feed waters likely to be encountered are already very high in metals, causing fouling issues, and $\mathrm{Ca}$ and $\mathrm{SO}_{4}$ resulting in $\mathrm{CaSO}_{4}$ scaling.

\subsection{Calcium Sulfate}

Calcium sulfate is made up of three major forms; anhydrite $\left(\mathrm{CaSO}_{4}\right)$, dihydrate $\left(\mathrm{CaSO}_{4} .2 \mathrm{H}_{2} \mathrm{O}\right)$ and hemihydrate $\left(\mathrm{CaSO}_{4} .0 .5 \mathrm{H}_{2} \mathrm{O}\right)$, with each having different solubilities. What makes $\mathrm{CaSO}_{4}$ different from other types of scale, such as $\mathrm{CaCO}_{3}$, is that it is $\mathrm{pH}$ independent and therefore scaling cannot be simply controlled by reducing the $\mathrm{pH}$ of the feed water $\left(\mathrm{CaSO}_{4}\right.$ solubility actually decreases in very acidic conditions). In order to try to prevent scaling, a suitable scale inhibitor should be dosed, in this case one which will prevent the formation of $\mathrm{CaSO}_{4}$ scale. Most current antiscalants work at a sub-stoichiometric level incorporating one or more closely inter-related mechanisms of threshold inhibition, crystal distortion and dispersion. Threshold inhibition prevents the precipitation of salts once the salt has exceeded its solubility product by blocking the attractive forces on concentrated anions and cations from forming protonuclei resulting 
in precipitation. Crystal distortion affects how the ions order themselves by causing an irregular shaping (distortion) of the crystal structure of the precipitated salt leading to a weak structure less likely to solidify on to surfaces. Dispersion occurs when the inhibitor chemisorbs itself onto the precipitated salt, imparting additional charge, causing repulsion, which ultimately leads to dispersion of solids and inhibition of organised crystal structure growth. If the correct antiscalant is chosen and the correct dose rate is used there should be minimal scaling of the membrane. However, when used incorrectly, the effect of $\mathrm{CaSO}_{4}$ scale can have a detrimental effect on the membranes within a $\mathrm{RO}$ plant to the point where irreversible damage is caused. The resulting scale has many different forms; $\mathrm{CaSO}_{4}$ initially occurs as needles and can transition into platelets and rosettes (Figures $1 \mathrm{a} \& b$ ). It is often found after membrane autopsy that $\mathrm{CaSO}_{4}$ forms on the feed spacer as well as on the membrane surface itself. These rosettes and platelets are often formed in the cross-over points of the feed spacer, where flow is at its lowest (Figure 1c). The scale can be damaging to the membrane surface through abrasion of these scaling particles, where the formed scale is pushed through the membrane by the pressure applied within the system, causing indents or even holes within the membrane surface. This will lead to a reduced salt rejection and increase in flux (more ions are able to pass through into permeate).
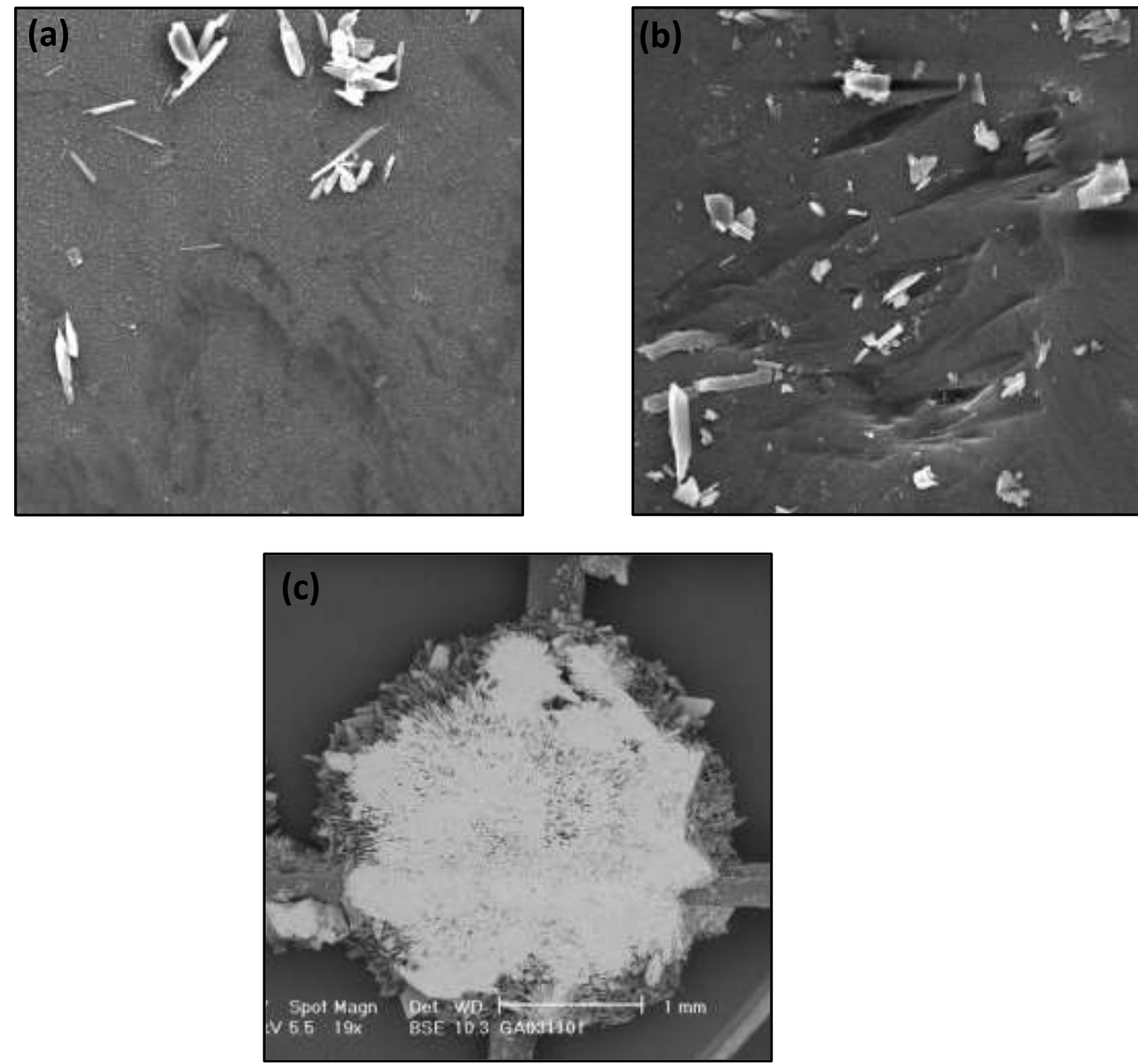

Fig. 1: (a) SEM photograph showing $\mathrm{CaSO}_{4}$ scale in needle form (b) as platelets that have damaged the membrane surface and (c) as rosettes formed on crossover point of vexar feed spacer.

\subsection{Methods of controlling Acid Mine Drainage}

Many different methods are currently used to combat AMD waters. The simplest approach is to prevent waste rock or tailings from coming into contact with any source of oxidation, therefore theoretically preventing oxidation from occurring. This is something that is virtually impossible to achieve and so more conventional methods used are to neutralise the AMD waters into other forms, or to collect the runoff to contain the acid, such as was the case with Iron Mountain in North Carolina (see Figure 2 which shows AMD tailings). The expense accumulated in the treatment of 
AMD waters is something that needs to be considered as well as the potential for disaster if treatment is not opted for. Currently the cheapest method of active water treatment is neutralisation using a base such as hydrated lime or sodium hydroxide. Although low in cost, this results in an increase in the TDS of the water, potentially such that it could damage aquatic life or human health (as seen in previous examples discussed earlier). A bigger issue is that it is a continuous process, or one that will never end and therefore in the long term will be a very expensive method of treatment.

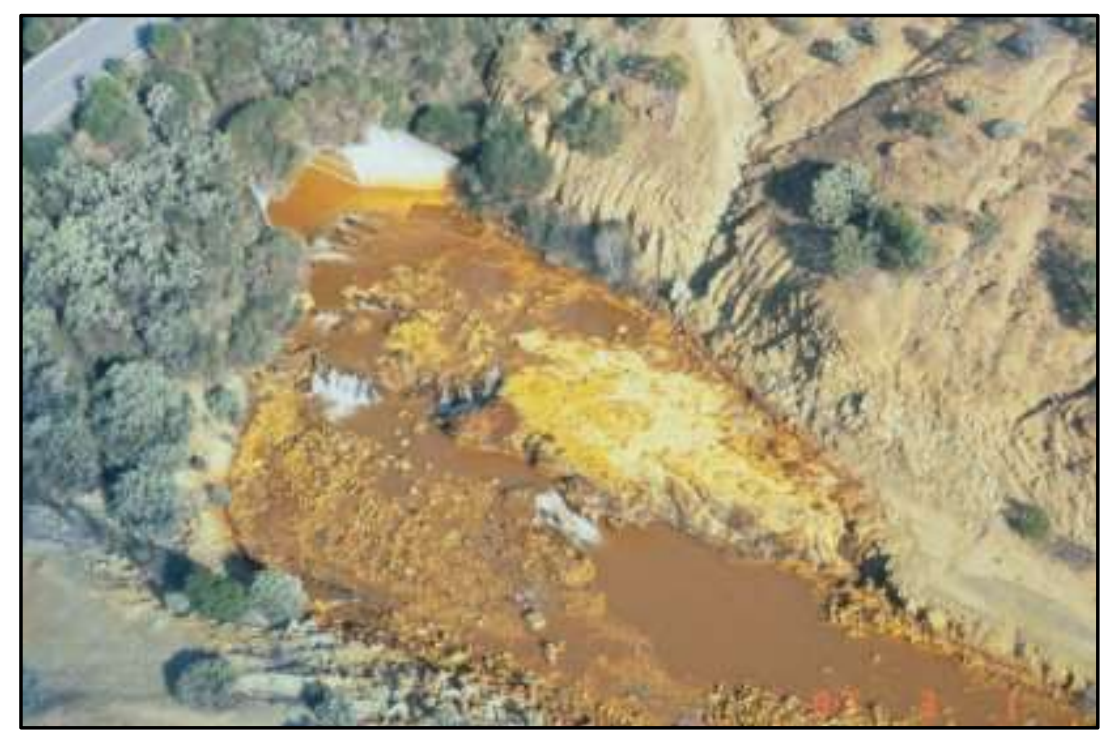

Fig. 2: AMD from mine tailings in Iron mountain, distinguishable by the rust colour of the water [3].

By implementing technology such as RO/UF, there is the potential to recover a certain percentage of these AMD waters for reuse onsite, for safe discharge and even for drinking purposes. The major difficulty arising from installing a RO plant with a mine is due to the harsh feed water conditions in the majority of AMD mines, the membranes would undergo rapid fouling and/or scaling. If the feed water is high in iron; which is typical of a mine with high amounts of pyrite, then this iron would have a dual effect, both fouling the membrane surface and acting as a catalyst in encouraging the onset of $\mathrm{CaSO}_{4}$ scale formation ( $\mathrm{SO}_{4}$ levels are high as a by-product of AMD). It would therefore be necessary to have an effective pre-treatment system as a minimum requirement within the RO plant in order to reduce or negate the potential for fouling and scaling. A suitable scale inhibitor would be needed to offset the potential for scaling and a clean in practice (CIP) protocol would be needed to minimise the risk of long-lasting damage to the membranes. Genmine AS26 is a low pH antiscalant that is particularly effective at decreasing the onset of $\mathrm{CaSO}_{4}$ scale formation in the presence of various metals, including iron, aluminium, copper and other metals typically found in a mine. The results for AS26 will be discussed in future papers.

This paper will discuss the ability to clean a membrane that has been scaled with $\mathrm{CaSO}_{4}$. In particular it will highlight the performance of some newly formulated cleaners, termed Cleaner A and Cleaner B with regards to cleaning $\mathrm{CaSO}_{4}$ scale. A membrane should never be allowed to get to the stage in where it has been irreversibly damaged by fouling/scaling; however this can happen unknowingly if the wrong antiscalant is used, the feed conditions change or even if there is simply human error when running the RO plant and the results can be very costly to replace each membrane. At the point where fouling/scaling is detected (should be a noticeable change in performance/quality of permeate produced), the plant should undergo a full clean in order to try to recover the operating conditions to what it was before fouling/scaling. If a loss in performance due to scaling is detected early enough there is the potential that the membranes would be salvageable, therefore avoiding the need to fully replace the membranes and subsequently saving a company a significant amount of money.

\section{Experimental Set-up}

All cleaning tests were carried out using a flat sheet test rig comprised of two mechanical stainless-steel plates that clamp a membrane coupon between them (Figures 3a), simulating that of a RO pressure vessel with the membrane, feed spacer and permeate spacer arranged in the same fashion as would be within a membrane. The stainless-steel component allows for the pressurisation of the system, with all tests in this paper run at $225 \mathrm{psi}$ ( $15 \mathrm{bar}$ ); typical conditions used in a brackish water system. The cleaning procedure itself is carried out using a beaker with cleaning solution that is 
recirculated for the appropriate time (usually 2-4 hrs) and flushed through with water after cleaning to remove any excess cleaner from the membrane. The size of the membranes tested on the flat sheet rig are usually $15 \mathrm{x} 15 \mathrm{~cm}\left(0.225 \mathrm{~m}^{2}\right)$.

As well as this there is also a flat sheet rig with a Perspex viewing window, enabling one to visually follow the progress/efficacy of the cleaning procedure (Figure $3 b$ ).

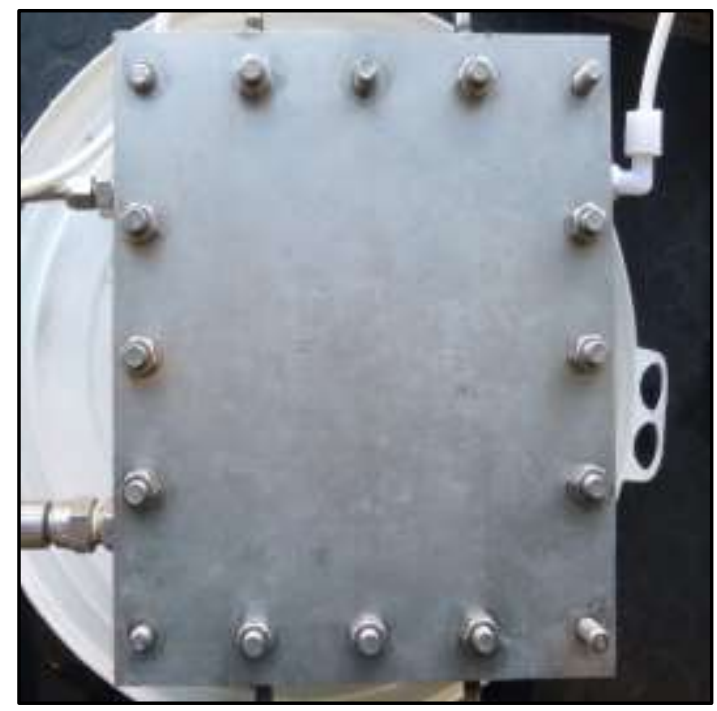

Fig. 3a: Stainless steel Flat Sheet Rig.

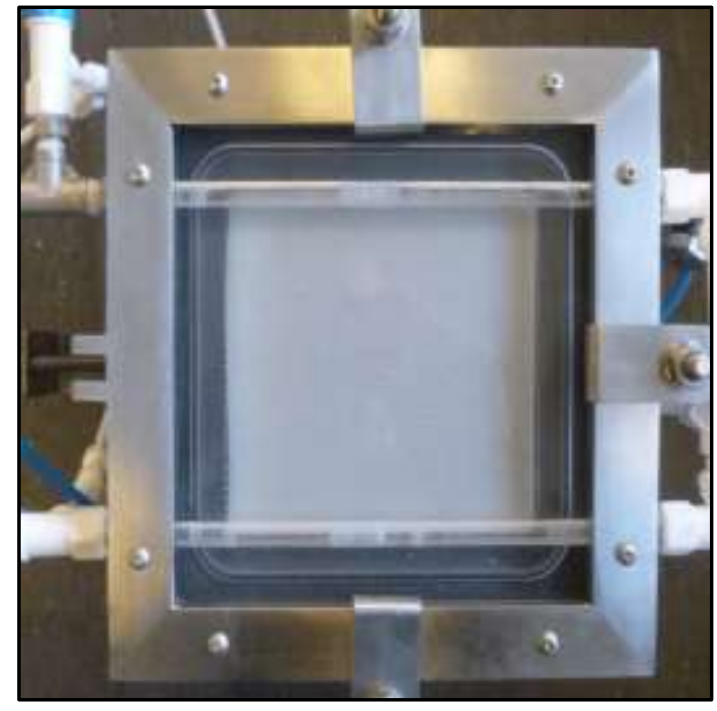

Fig. 3b: Flat Sheet Rig with viewing window.

\section{Results and Discussion}

This paper focused on the cleaning of $\mathrm{CaSO}_{4}$ from a membrane, particularly from the vexar feed spacer which can accumulate large amounts of $\mathrm{CaSO}_{4}$ scale within a full membrane unit. Various cleaners (Cleaners A-D) were used to primarily test if $\mathrm{CaSO}_{4}$ could be effectively removed and therefore which cleaner was the most efficient.

\section{Flat sheet cleaning and characterization protocol used}

The standard operating conditions used for these experiments where:

- A flux rate was measured at standard operating conditions for the membrane type ( $2000 \mathrm{ppm} \mathrm{NaCl}$ solution at $225 \mathrm{psi}$ ). The recirculation rate was $1000 \mathrm{~mL} / \mathrm{min}$ and the temperature of each was normalized to $25^{\circ} \mathrm{C}$.

- Cleaning solutions were made up as $5 \%$ in water and recirculated at 40 psi for 3 hours. Within this time period the cleaning procedure was a 30 minute circulation of cleaner followed by a 15 minute soaking period, repeated over the test period.

- After cleaning the flux rate and SR are again measured to see if there is any improvement in performance.

\subsection{Cleaning Results- Feed Spacer}

The following cleans were carried out on heavily scaled $\mathrm{CaSO}_{4}$ membrane and vexar spacer:

Clean 1: $5 \%$ Cleaner A at $\mathrm{pH}=11.9$ for 3 hours @20-25 ${ }^{\circ} \mathrm{C}$.

Clean 2: $5 \%$ Cleaner B at $\mathrm{pH}=11.3$ for 3 hours @20-25 ${ }^{\circ} \mathrm{C}$.

Clean 3: $5 \%$ Cleaner $\mathrm{C}$ at $\mathrm{pH}=7.0$ for 3 hours @ 20-25 ${ }^{\circ} \mathrm{C}$.

Clean 4: $5 \%$ Cleaner D at pH=11.8 for 3 hours @20-25 ${ }^{\circ} \mathrm{C}$.

The results for each of these tests can be found in the table below: 
Table 1: Results of different cleaning tests on $\mathrm{CaSO}_{4}$ scale.

\begin{tabular}{|c|c|c|c|c|c|c|}
\hline & \multicolumn{2}{|c|}{ Flux rate (LMH) @ 25 ${ }^{\circ} \mathbf{C}$} & \multicolumn{2}{c|}{ \% Salt Rejection } & \multicolumn{2}{c|}{ Weight of Spacer (g) } \\
\cline { 2 - 7 } & $\begin{array}{c}\text { Before } \\
\text { Clean }\end{array}$ & After Clean & $\begin{array}{c}\text { Before } \\
\text { Clean }\end{array}$ & After Clean & $\begin{array}{c}\text { Before } \\
\text { Clean }\end{array}$ & After Clean \\
\hline Clean 1 & 58.5 & $\begin{array}{c}54.9 \\
(-6.2 \%)\end{array}$ & 69.5 & 77.9 & 32.7 & 2.5 \\
\hline Clean 2 & 63.1 & $\begin{array}{c}51.1 \\
(-19.0 \%)\end{array}$ & 76.4 & 76.0 & 31.6 & 1.6 \\
\hline Clean 3 & 60.1 & $\begin{array}{c}67.1 \\
(+11.6 \%)\end{array}$ & 77.9 & 74.6 & 31.3 & 3.4 \\
\hline Clean 4 & 64.8 & $\begin{array}{c}65.2 \\
(+0.6 \%)\end{array}$ & 67.7 & 75.0 & 29.4 & 15.7 \\
\hline
\end{tabular}

Flux rate was tested with $2000 \mathrm{ppm} \mathrm{NaCl}$ at $225 \mathrm{psi}$

In Table 1 the flux and SR values can be misleading, as although in each test differing amounts of $\mathrm{CaSO}_{4}$ were removed, the flux values actually show a decline (flux should increase). This is most likely due to the nature of the test, with the layer of $\mathrm{CaSO}_{4}$ being so thick on the feed spacer that the rig was prone to leaking. This amount of scale also made controlling the pressure difficult initially, with the system becoming more stable after cleaning. Due to this uncertainty, it is better to focus on the weight of spacer column as these gave a more quantitative picture of what was happening with each clean (Figure 4) and how much $\mathrm{CaSO}_{4}$ is being removed.

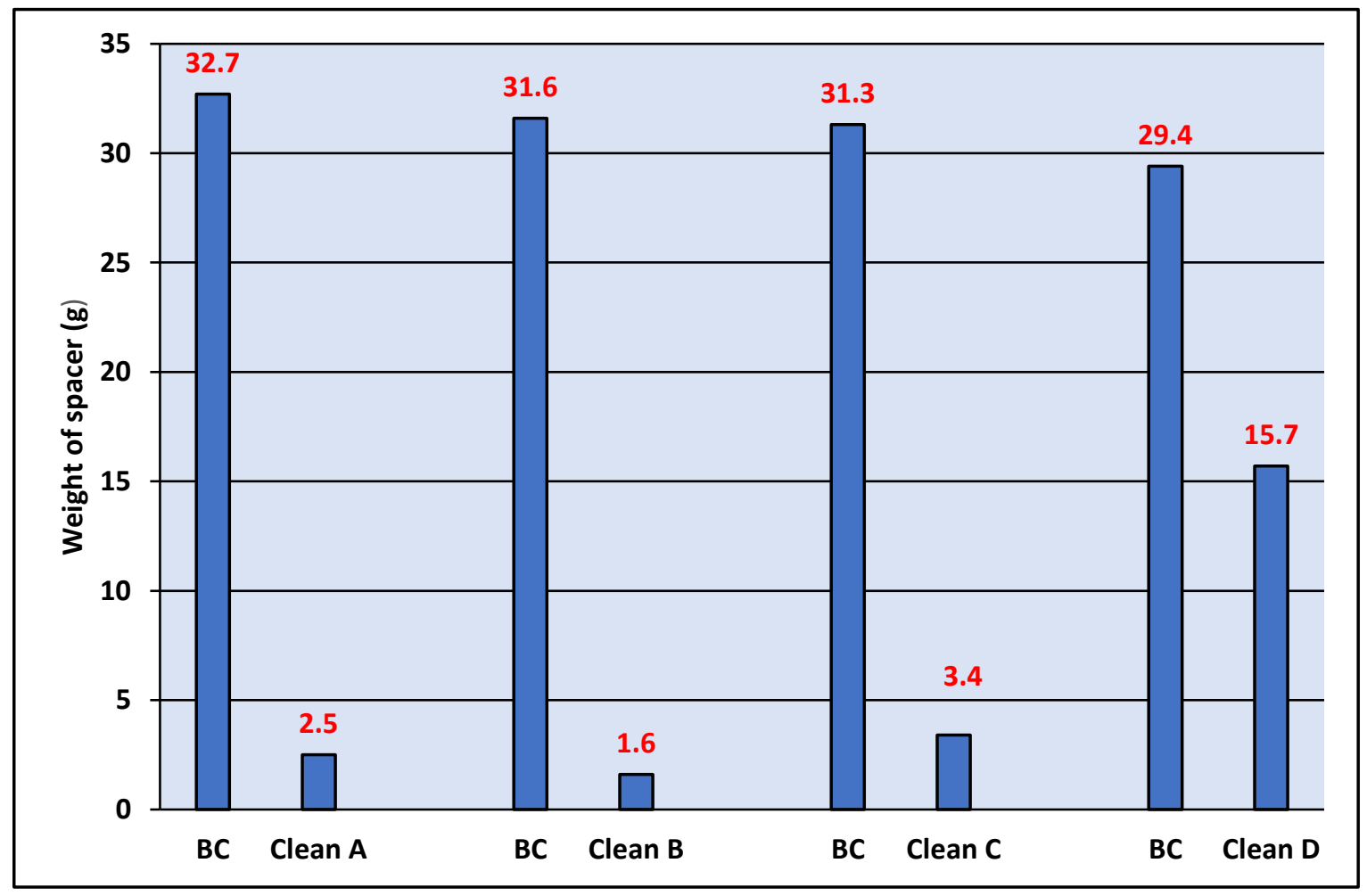

Fig. 4: Graph showing weights of spacer after cleaning.

\section{2. $\mathrm{CaSO}_{4}$ cleaning pictures- Feed Spacer}

The cleaning pictures below give an insight to the level of $\mathrm{CaSO}_{4}$ scale formation on the vexar spacer. 


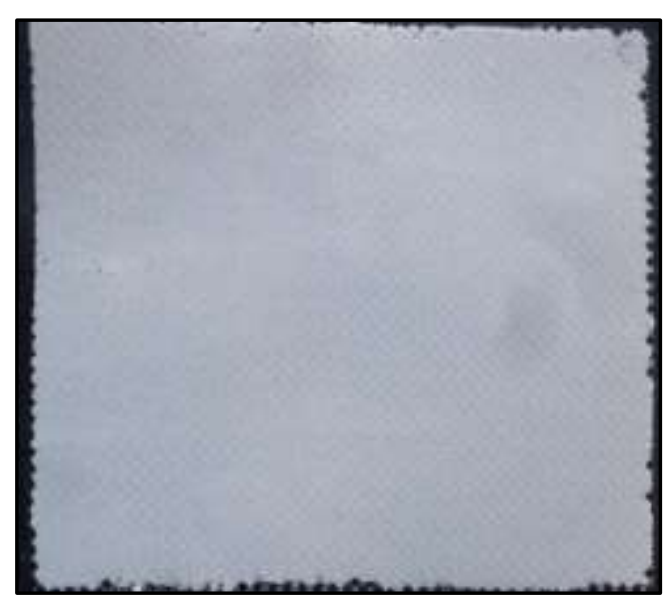

Fig. 5: Vexar spacer heavily scaled with $\mathrm{CaSO}_{4}$

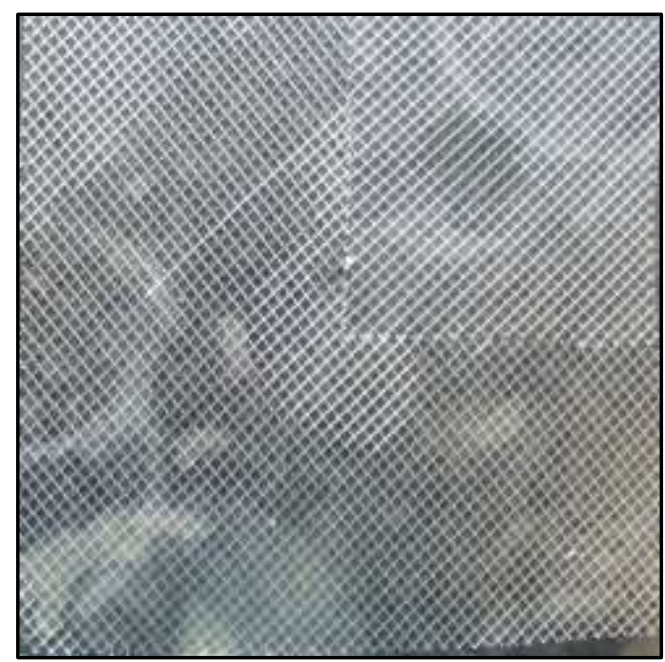

Fig. 7: Clean 2.

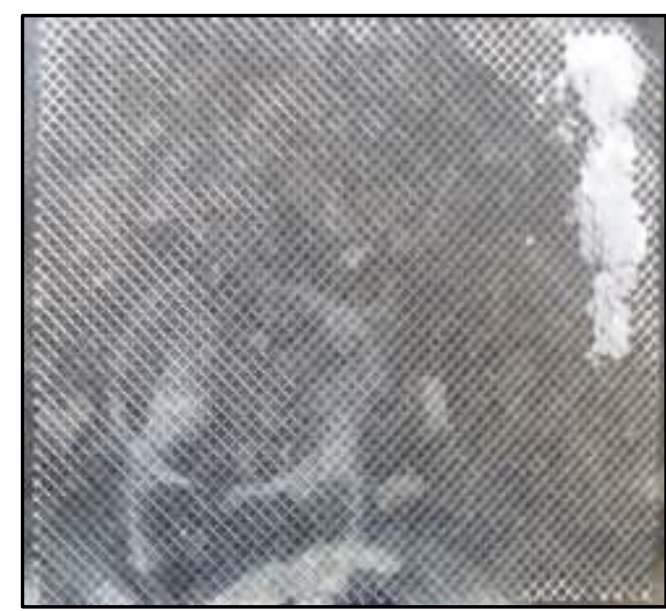

Fig. 6: Clean 1.

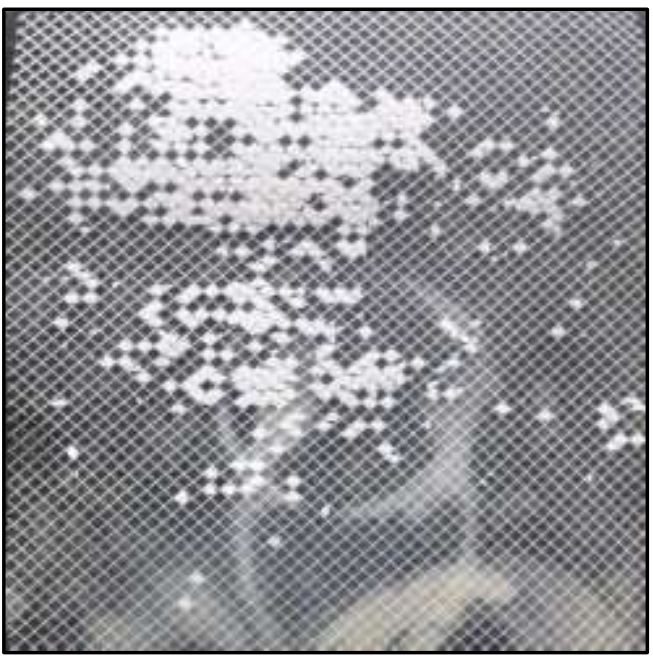

Fig 8: Clean 3.

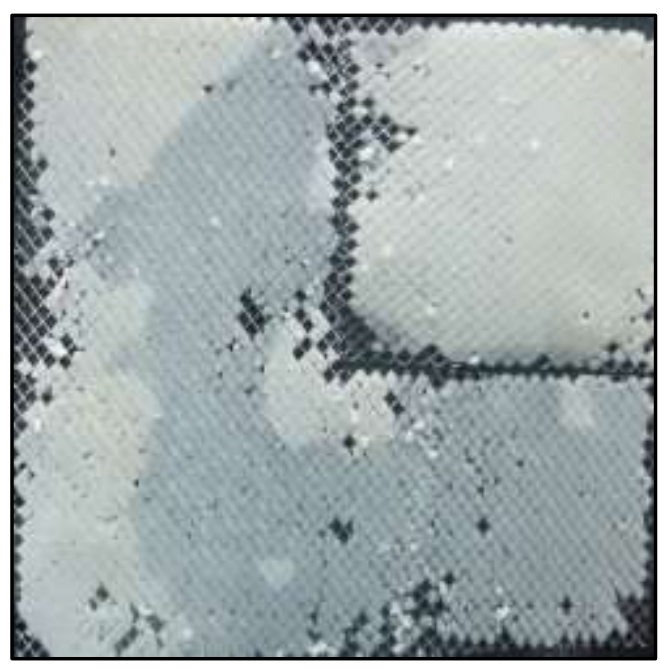

Fig. 9: Clean 4. 
All of the above pictures give a visual representation of the initial level of scaling on the feed spacer (Figure 5) as well as the performance of each clean.

\subsection{Evaluation of results}

Of the results seen in Table 1, the best method of quantifying the cleaning ability of each cleaner was to determine the amount of $\mathrm{CaSO}_{4}$ removed from the vexar spacer. This was done quantitatively, by weight. From Table 1, it is seen that Cleaner A and Cleaner B removed the majority of $\mathrm{CaSO}_{4}$ scale; 96.5 and $99.3 \%$ respectively. Cleaner C is slightly less effective at $93.3 \%$ and Cleaner D gave the worst result with $48.9 \% \mathrm{CaSO}_{4}$ scale removed. Due to the thickness of scale on both spacer and membrane, the flat sheet rig was prone to leaking and thus the pressure was difficult to stabilize, therefore flux and SR values are less important in this instance with weight loss being much more accurate, as well as how the spacer looked to the naked eye and under a microscope.

\section{4. $\mathrm{CaSO}_{4}$ scaling as seen under the microscope}

Although visually Figures 5-9 gave a good indication of the removal of $\mathrm{CaSO}_{4}$ scale, further analysis under microscopic conditions gives additional insight into how much of the scale has been removed. In the figures below (Figures 10-11), it can be seen how heavily scaled the spacer was initially (Figure 10) and how much of the scale had been removed after a 3-hour clean (Figure 11):

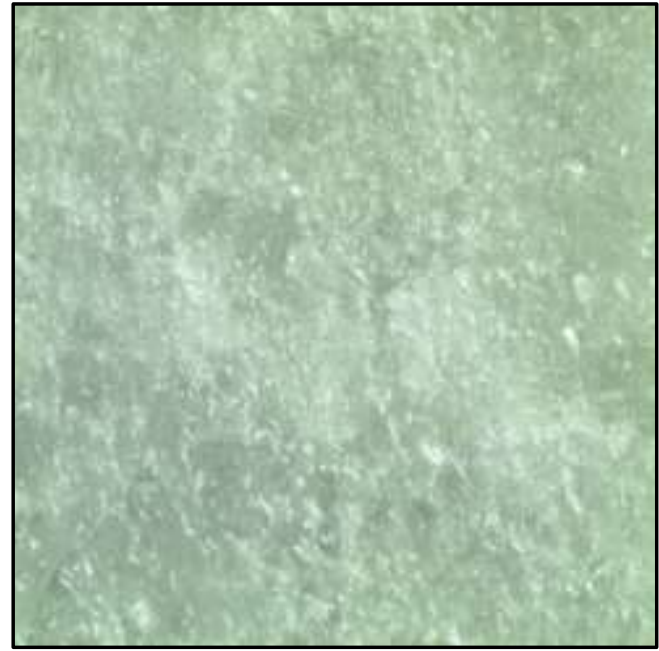

Fig.10: Spacer before cleaning.

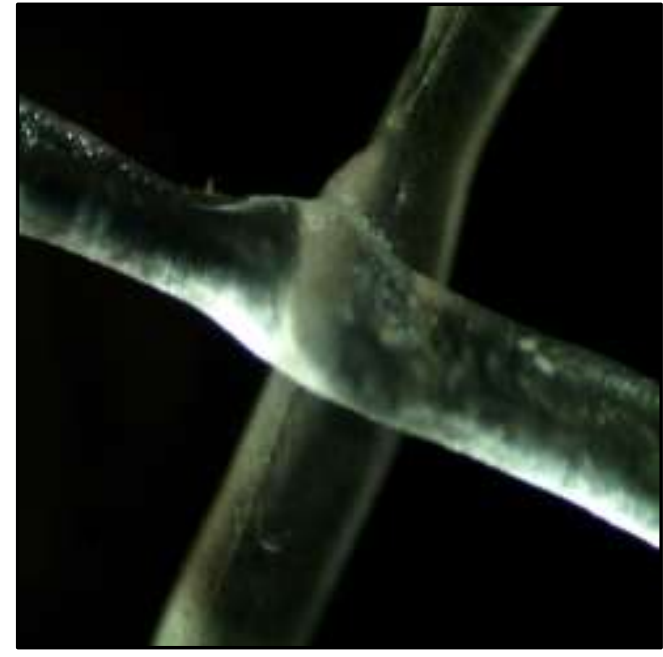

Fig. 11: Spacer after 3-hour clean with Cleaner A.

\subsection{Infra-Red Spectroscopy/ATR}

Infra-Red spectroscopy is a useful analytical method used to help with the identification of different functional groups within chemical compounds. In the application of membrane technology, it is a useful tool in identifying the chemical composition of the membrane, particularly the polyamide and polysulfone layers that make up the majority of conventional membranes. It is particularly adept at providing information regarding the identification of surface foulants/scaling species as well as whether or not the polyamide layer has been compromised in some way as a result of poor upkeep of the RO plant.

Attenuated Total Reflectance (ATR) is used as a physical aid in tandem with IR spectroscopy with regards to sample preparation. One of the drawbacks of IR spectroscopy is the preparation time needed and the reproducibility of the sample. With ATR this problem is negated as no preparation is needed, meaning that samples can be run in a few minutes, giving spectra that is more accurate than IR alone. This is because the technique itself is more sensitive due to an increased signal to noise ratio within the spectrometer and an ATR crystal with a high refractive index.

In all of the above cleans discussed above, $\mathrm{CaSO}_{4}$ was identified as the main scaling species detected. This can be further confirmed by measuring the $\mathrm{CaSO}_{4}$ by ATR-IR (Figure 12). 


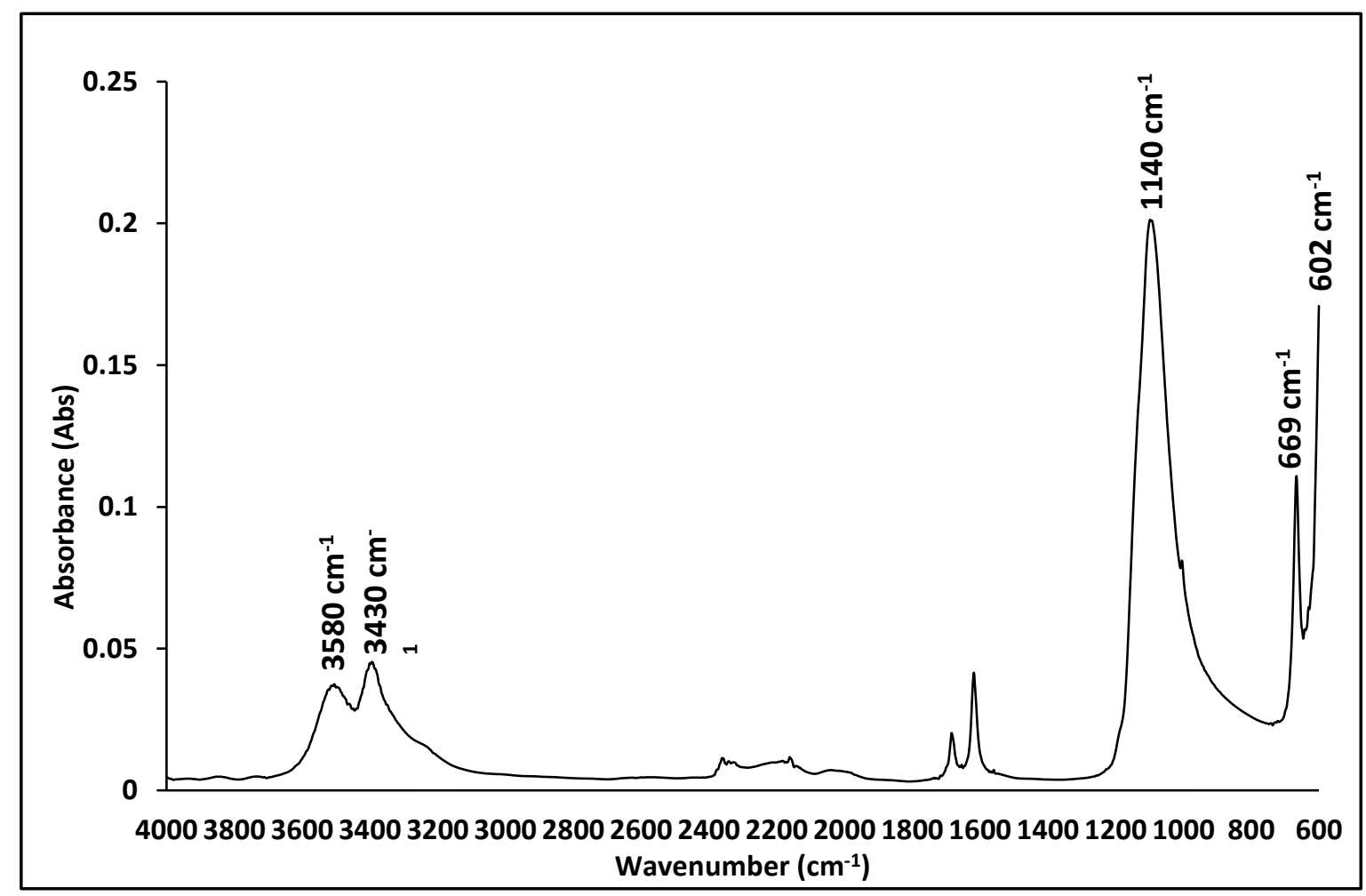

Fig. 12: Infra-Red spectrum showing $\mathrm{CaSO}_{4}$ scaling (gypsum) across the membrane/feed spacer.

The ATR-IR spectrum above shows that for $\mathrm{CaSO}_{4}$ with the peaks around $1140 \mathrm{~cm}^{-1}$ (which can split into two peaks at 1116 and $1146 \mathrm{~cm}^{-1}$ ) and the peaks at 669 and $602 \mathrm{~cm}^{-1}$ are assigned of the bending and stretching modes of the sulfate anion [4]. The stretching vibrations for $\mathrm{H}_{2} \mathrm{O}$ can be assigned to the peaks at 3580 and $3430 \mathrm{~cm}^{-1}$ for gypsum. If the $\mathrm{CaSO}_{4}$ scale is present on the membrane it is also useful to compare the spectrum of a membrane that has been cleaned (Figure 13) to highlight the difference between them and elucidate the efficacy of the clean.

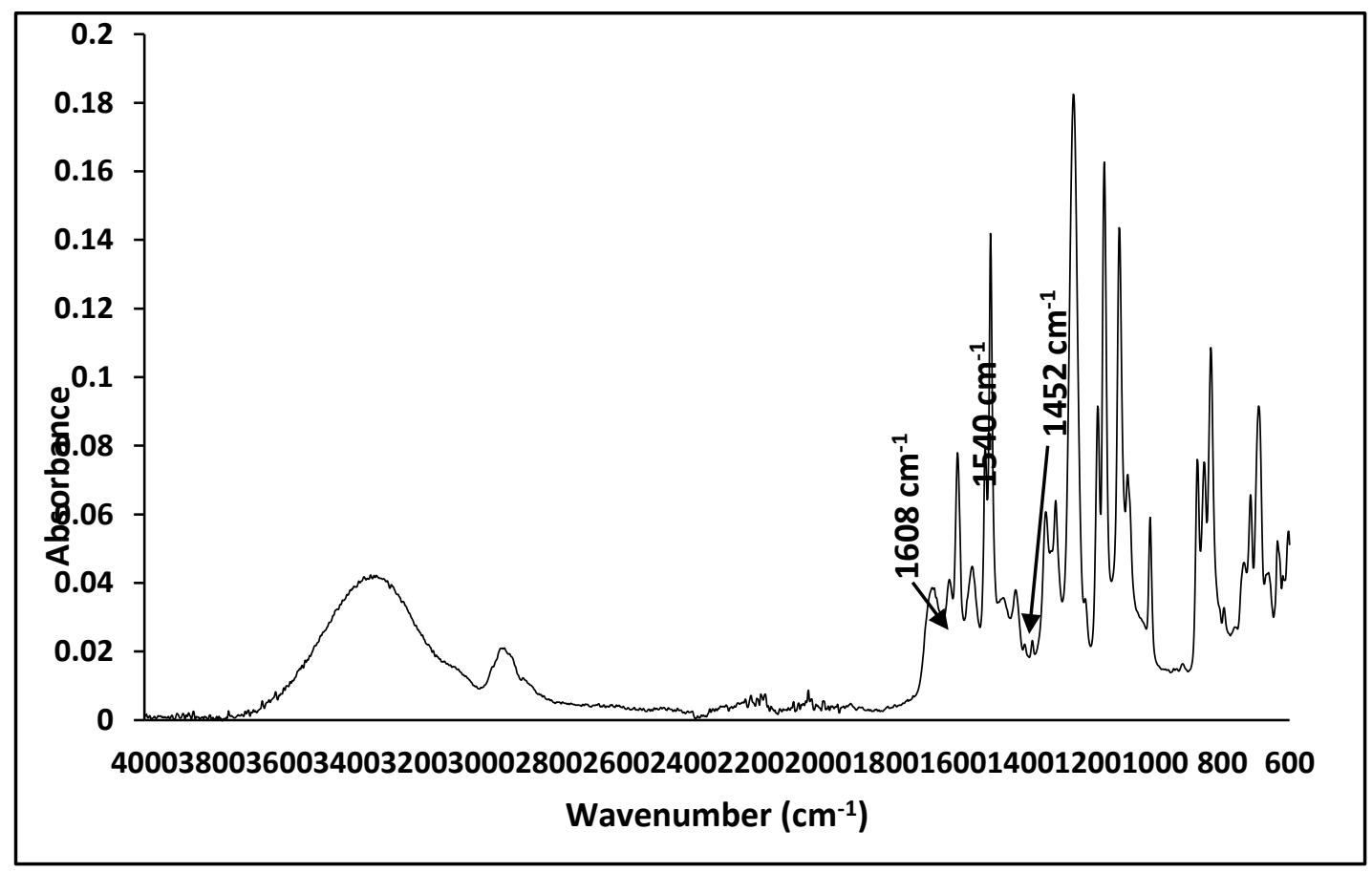

Fig. 13: Infra-Red spectrum showing the membrane surface after Cleaner B. 
The typical configuration of a membrane is made up of a thin layer of polyamide (this is the active layer) and a much broader polysulfone support layer. The peaks labelled at 1608,1540 and $1452 \mathrm{~cm}^{-1}$ are typical of the polyamide bands, with the other peaks in this region $\left(1600-1000 \mathrm{~cm}^{-1}\right)$ are attributed to the polysulfone layer, specifically the peaks at 1244 , 1585,1020 and $830 \mathrm{~cm}^{-1}$. If a membrane is fouled/scaled the IR-ATR spectrum will be very different (Figure 12) with these bands not visible due to the fouling/scaling species present on the surface. In comparing Figures 12 and 13, it can be seen that the $\mathrm{SO}_{4}$ peak present in Figure 12 is no longer visible in Figure 13. This shows that the $\mathrm{CaSO}_{4}$ has been effectively removed as the membrane peaks are now detected, in particular the peaks at 1608, 1540 and $1452 \mathrm{~cm}^{-1}$, those of the polyamide layer.

\section{Conclusions}

There has been much discussed within the literature regarding the need for a change in behaviour regarding the safe disposal of run-off from mining operations, with storage no longer a viable option. Current methods such as neutralizing these AMD waters is not seen as a solution for the issue but more of a continuous treatment to an ongoing problem. In the past number of decades there have been many catastrophes worldwide as a result of mining relating incidents, including the contamination of surrounding rivers as a result of both human error and failed infrastructure. In the worst cases, loss of life through the collapse of dams, drinking water has become contaminated; as well as loss of wildlife is often too common an occurrence, with the thought being that one catastrophe is one too much. As well as this, large volumes of water are needed to leach precious metals out of their ores; in a world where potable water is becoming more sought after, it is inevitable that such practices can no longer continue unless the waste from the extraction process can be recovered and reused.

Although desalination and in particular RO technologies is not a new technology, the use of such within the mining industry is a relatively new phenomenon. The first plant to recover metals using RO membranes was not operational until the 1990's. Since then its growth has been a slow one, with operational costs, a lack of published material and secrecy from the mining industries resulting in much slower growth than first anticipated. Membrane prices have reduced significantly since their inception, with RO technologies seen as an alternative to current methods used.

As with any waste water stream, there are numerous different suspended and dissolved solids, which is only increased when dealing with mine water waste streams, particularly those that are low $\mathrm{pH}$. This makes designing a RO plant more difficult as fouling and scaling tends to occur, and in particular $\mathrm{CaSO}_{4}$. The authors have focused on designing new cleaners and antiscalants that are specifically targeted towards mining waters.

In all tests performed, $\mathrm{CaSO}_{4}$ scale was removed to various different levels, with Cleaner $\mathrm{A}$ and Cleaner $\mathrm{B}$ proving best, closely followed by Cleaner $\mathrm{C}$. It should also be noted that $\mathrm{CaSO}_{4}$ scale is difficult to clean, and its formation can damage a membrane if proper pre-treatment/antiscalant control is not used. This paper does show that if a plant has a problem with $\mathrm{CaSO}_{4}$ scale, it is possible to clean, however this must happen as soon as scaling is detected before any irreversible damage is done. It is always best to prevent the formation of scale rather than treat scale, therefore pretreatment should be considered when designing a plant. In this instance effective pre-treatment would involve using a suitable low $\mathrm{pH}$ antiscalant, such as Genmine AS26, at the correct dose rate based on the feed conditions. In some cases, this is still not enough as feed conditions can suddenly change and so cleaning is sometimes the only option left to prevent costly and at times irreversible damage to the membranes.

\section{References}

[1] P. Szyplinska, "Thirsty world of mining: Harvesting new water solutions," 2012.

[2] A. Akcil, S. Koldas, "Acid Mine Drainage (AMD): causes, treatment and case studies," Journal of Cleaner Production, vol. 14, pp. 1139-1145, 2006.

[3] NOAA Restoration Center \& Damage Assessment and Restoration Program. [Online]. Available: https://www.photolib.noaa.gov/htmls/r00imm60.htm

[4] H. Boke, S. Akkurt, S. Ozdemir, E. H. Gokturk, E. N. Saltik, "Quantification of $\mathrm{CaCO}_{3}-\mathrm{CaSO}_{3} \cdot 0.5 \mathrm{H}_{2} \mathrm{O}-$ $\mathrm{CaSO}_{4} \cdot 2 \mathrm{H}_{2} \mathrm{O}$ mixtures by FTIR analysis and its ANN model," Material Letters, vol. 58, 723-726, 2004. 